\title{
Demographic representation of COVID-19 social media and information engagement in Nigeria
}

\author{
Winifred Ekezie ${ }^{1}$, Genevieve Bosah ${ }^{2}$
}

\section{AFFILIATION}

1 Diabetes Research Centre, University of Leicester, United Kingdom

2 Department of Media, University of Hertfordshire, United Kingdom

\section{CORRESPONDENCE TO}

Winifred Ekezie. Diabetes Research Centre, University of Leicester, Leicester General Hospital, Gwendolen Road, Leicester, LE5 4PW, United

Kingdom. E-mail: wce2@leicester.ac.uk
ORCID ID: https://orcid.org/0000-0001-6622-0784

KEYWORDS

Nigeria, COVID-19, information, communication, vaccine, social media

Received: 18 December 2020, Revised: 17 April 2021,

Accepted: 13 May 2021

https://doi.org/10.18332/popmed/137087

\begin{abstract}
INTRODUCTION Disease outbreaks affect demographic groups differently, as evident with the COVID-19 pandemic. Public health communication during these periods, especially on social media, aims to encourage individuals to improve their health by practicing healthy behaviors. However, the management of the COVID-19 pandemic has been significantly influenced by misinformation. This study aimed to understand how selected demographic factors in Nigeria influenced engagement with COVID-19 pandemic information on social media.

METHODS A cross-sectional survey was conducted in June 2020. Data on demography, information source and media consumption patterns, and information accuracy and responsibility were collected through snowballing technique and promotions through emails and social networking sites. RESULTS A total of 1127 respondents participated, 59\% male, $85 \%$ aged $18-45$ years, and $92.1 \%$ had an undergraduate degree or above. Most participants used social media,
\end{abstract}

especially Twitter (39.4\%). About 93.1\% received misinformation about COVID-19, 82.9\% believed social media were the primary source of inaccurate information, while $59.7 \%$ took action to verify the accuracy of the information received on protection. Most preferred Twitter, while older people preferred WhatsApp, and those with the highest education used both platforms evenly. Younger people considered international organizations most responsible for information accuracy but also highlighted individuals were also responsible.

CONCLUSIONS Similar perceptions and engagement with COVID-19 pandemic information were observed across different demographic groups in Nigeria, with all groups relying significantly on social media. To ensure the availability of accurate information and appropriate responses to COVID-19 management, more meaningful social media engagement is required to reduce the risk of harmful misinformation, particularly related to vaccination.

protecting self and others; individuals looked up these guidelines and tips for themselves and shared them with others. With large amounts of unchecked user-generated contents on social media, the risk of misinformation increases as media consumption patterns change misinformation levels ${ }^{3}$. Hence, with an increase in the number of social media platforms and the volume of usergenerated content, the risk of running into misinformation is unavoidable.

Social media engagement has fast emerged as a complement for supporting existing evidence and best practices. Social media are participatory and reciprocal and can target and reach diverse audiences, allowing the creation 
of platforms for listening and collecting feedback in realtime whilst permitting increased direct engagement ${ }^{4}$. The use of social media may have significantly increased during the COVID-19 global crisis because of its capacity to engage audiences in multi-way conversations and interactions. However, during disease outbreaks, misleading posts are often more popular than accurate and relevant public health information, thereby presenting conflicting messages 5 . Another unintended consequence of social media is the shift in information evaluation burden from professional gatekeepers to individual consumers ${ }^{6}$. Hence, individuals play a significant role in the creation, production, distribution and re-production of misinformation.

Globally, the management of the COVID-19 pandemic has been potentially influenced by misinformation. Many people used unconventional protection approaches in Nigeria, including herbal remedies and the antimalarial drug, hydroxychloroquine, after false messages spread through social media ${ }^{7}$, leading to cases of overdose, hospitalization and death ${ }^{8}$. Social media engagement needs to be studied better to determine how to encourage healthy behaviors through these channels. However, research on social media and public health communication in developing countries, especially Africa, is limited ${ }^{9}$. Although literature exists on information dissemination related to the pandemic, there are gaps in the demographic attitudes. Evidence has shown that sociodemographic factors, such as age, gender, education level, and ethnicity, play a significant role in the self-protecting behaviors during the COVID-19 pandemic ${ }^{10}$. Consequently, understanding the relationship between population attributes and their actions would be critical for misinformation management and public health message development, particularly vaccine communication, as social media offline actions often predict vaccination belief ${ }^{11}$. This research aimed to understand how selected demographic factors in Nigeria influenced engagement with COVID-19 pandemic information on social media.

\section{METHODS}

A cross-sectional online survey, using Google Form, was used to collect data related to the spread of COVID-19 information in June 2020. Eligible participants were adults aged $\geq 18$ years residing in Nigeria. Data on demography, information source, media consumption patterns, and information accuracy and responsibility were collected through snowballing technique and promoted through emails and social networking sites. Participation was voluntary, and no incentives were offered. Responses were treated as categorical variables and analyzed using descriptive statistics to explore the relationship between demographic factors and social media information source, dissemination, responsibility, and accuracy checks. Analysis was performed using Stata 16 statistical software. Ethical approval was received from the University of Hertfordshire (HUM/SF/ $\mathrm{UH} / 04647)$.

\section{RESULTS}

There were 1127 respondents, 59\% (665/1127) were males, $85.1 \%(959 / 1127)$ were aged $18-45$ years, and $92.1 \%$ $(1029 / 1127)$ had an undergraduate degree or above. Almost all respondents used social media as a primary source of information about the COVID-19 pandemic, and the most helpful platform was Twitter (39.4\%, 444/1127) (Table 1). Most participants received speculative information on protection against COVID-19 through social media platforms, for example, the use of chloroquine, hot water, bleach, and herbal remedies. About 59.7\% (633/1061) checked the validity of the information received on authorized platforms, such as the World Health Organization website. In comparison, $12.3 \%(130 / 1061)$ shared the information with their social contacts without verification. Overall, $93.1 \%$ (1049/1127) received misinformation about the pandemic through social media, and 82.9\% (934/1127) believed social media was the primary source of inaccurate information. However, only $23.4 \%$ (262/1120) emphasized individuals had a responsibility in news consumption compared to $71.2 \%(797 / 1120)$ and $61 \%(683 / 1120)$ who stated national governments and international agencies, respectively, were responsible for managing fact-checking and verification of information related to the pandemic.

Trends in perception and attitudes toward social media information management were similar across age groups, gender, and education level. However, older people preferred WhatsApp to Twitter, while those with the highest education qualification used both platforms evenly. All groups reported checking credible sources to verify information received, but the only respondent in the lowest education category tried out some recommendations and considered social media information accurate. Some respondents also considered information from sources that usually carried correct news to be accurate without checking personally $(22.2 \%, 248 / 1118)$. Those aged 18-25 years mostly believed international institutions were responsible for information accuracy, but the consensus was that national governments were the most responsible. There was also significant emphasis on the responsibility of community leaders, social media developers, and influencers, while opinions of individual responsibility decreased as age increased from 24-35 years to 60 years.

\section{DISCUSSION}

Social media platforms allowed unprecedented access to COVID-19 public health messages in Nigeria. Twitter was the most common social platform, especially among younger age groups. Information on COVID-19 prevention was shared widely; most users made efforts to check their validity, but most considered national and international organizations more responsible for information accuracy. Similar patterns of social media information use and attitudes in Nigeria have been observed in another study on knowledge, risk perceptions and precautionary behaviour ${ }^{12}$. 
Table 1. Participant demographic information preference and perceptions

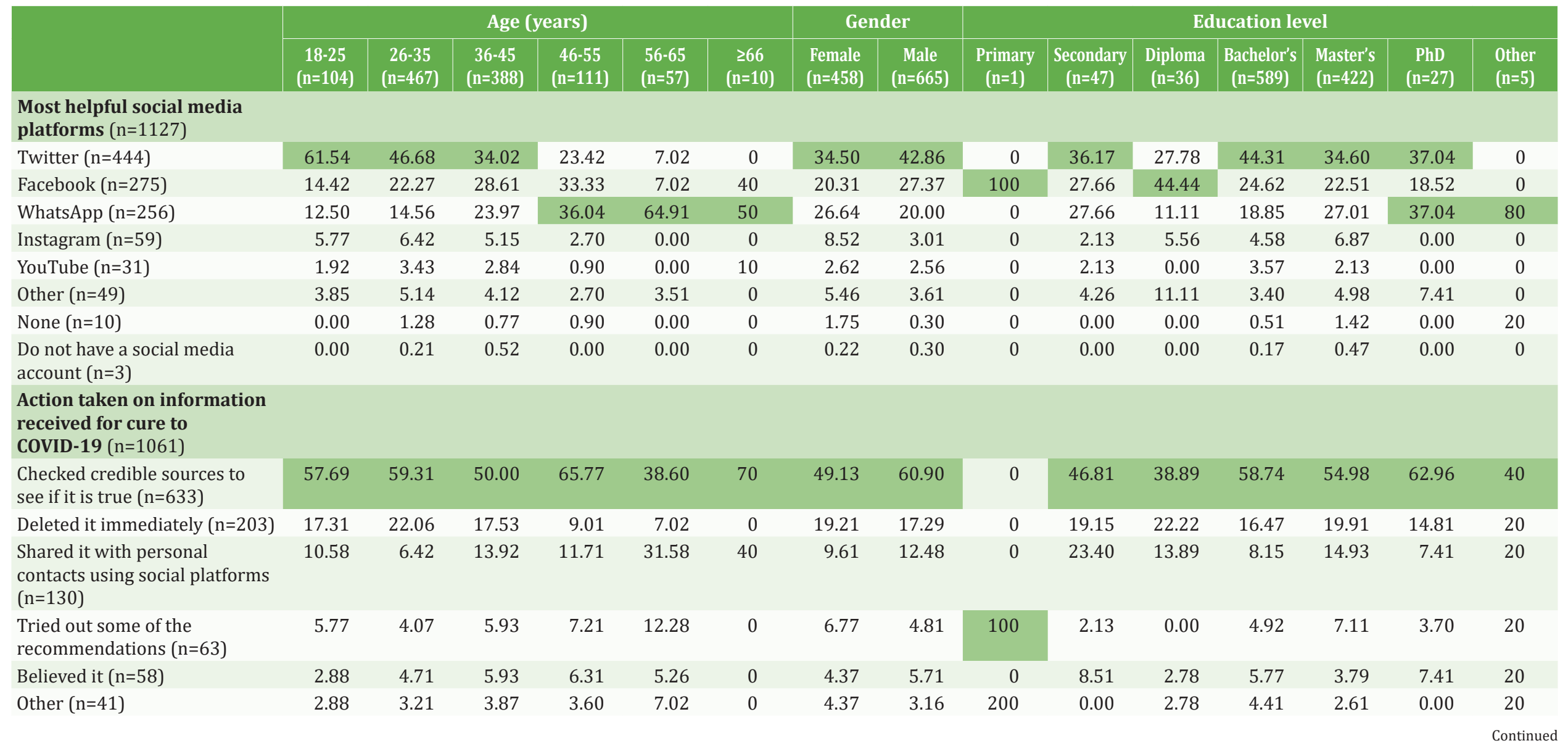




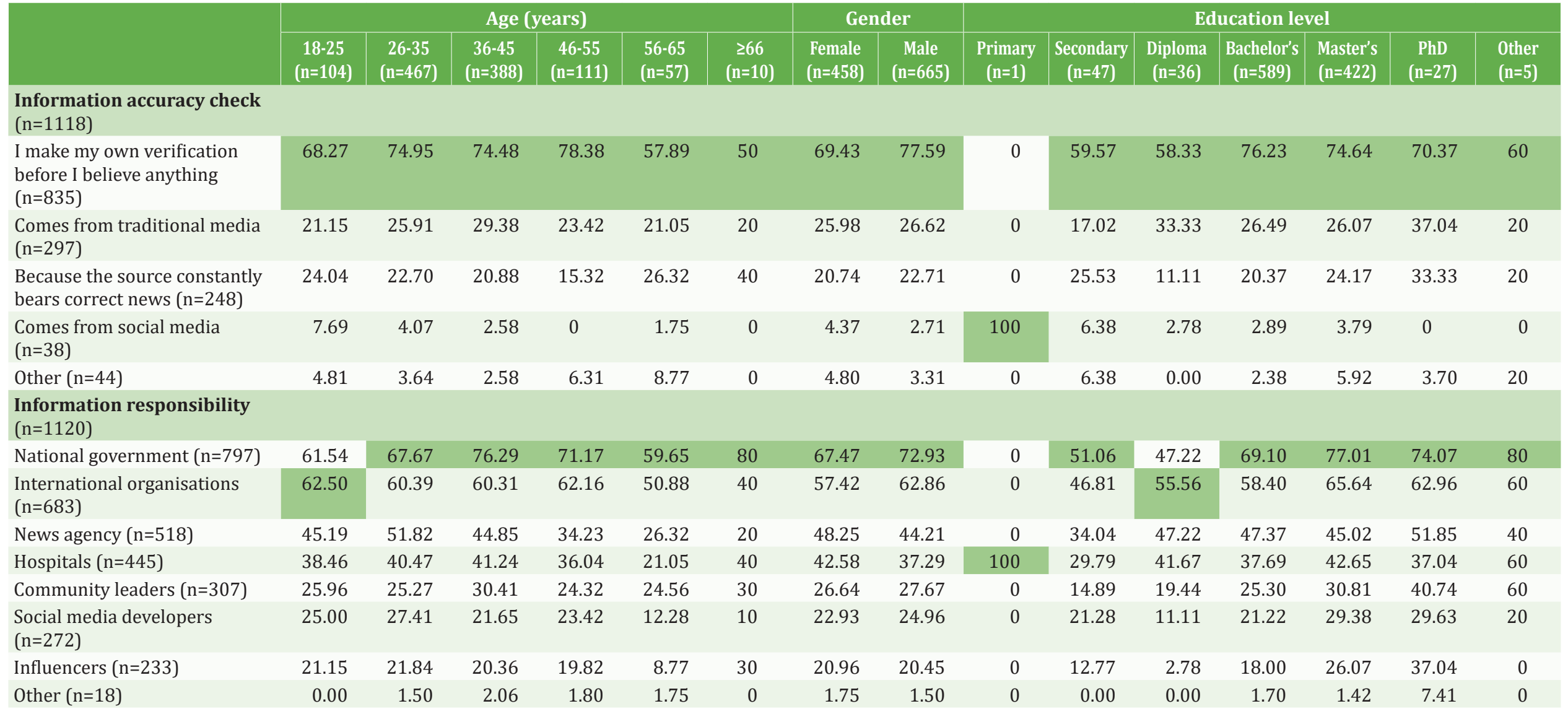

Highlighted boxes show the options with the highest responses among each group. 
Although response variations among the different demographic groups were not wide, experience from past outbreaks shows the importance of incorporating demographic analysis into preparedness and response efforts to improve health interventions effectiveness and promote gender equality and health equity ${ }^{13}$. This point is also critical for managing public health social media engagement challenges, which include loss of message control, shift to 'direct-to-consumer engagement', and public health outcome impact that could skip certain groups if they are not explicitly highlighted ${ }^{14}$.

Dependence on social media as a primary information source, fear of COVID-19 infection and weak government responses in Nigeria escalated the circulation of fake news ${ }^{15}$. There is a need to ensure that responses to protection against COVID-19 do not perpetuate harmful norms, such as vaccine hesitancy due to misinformation ${ }^{3}$. As respondents in this study were open to seeking information, and education appeared to influence social media information attitudes, it is vital to support meaningful media verification engagement using the media platform preferences of different groups. Such engagement needs to include individual networks and organizations to ensure efforts and responses are not discriminated against ${ }^{4}$. This could include awareness campaigns and information dissemination interventions presented through public organizations' online and in-person services, such as libraries and academic institutions, which have existing public information platforms and the ability to validate information ${ }^{15}$.

For effective control of the pandemic, COVID-19 vaccine programs, group prioritization and communication are vital. It is necessary to deliver information through various sources and target different groups to ensure wider engagement and disinformation reduction. Having adequate knowledge of COVID-19 improves precautionary behavior; hence, targeted messages could motivate individual precautionary behaviors and practices ${ }^{12}$. To ensure vulnerable groups are reached, effective communication through preferred media platforms is essential. This approach would provide accurate and supportive care messages needed to enhance personal safety. Achieving this requires leveraging social media for community engagement through actively listening, monitoring, and responding to social media conversations; creating opportunities for users to engage with health organizations; welcoming solicited user-generated content; incorporating voices of credible influencers in the front lines of social responses, policies or practices; and ensuring diverse representation in national and local COVID-19 policy spaces ${ }^{14}$.

Public health communication has different engagement levels, which all carry potential risks, benefits, and challenges when embracing social components ${ }^{13}$, but using social media works best when integrated with traditional public health communication channels. Nevertheless, the multidimensional nature of social media shows that credible public health communication is critical for infectious diseases monitoring and rapid response deployment; hence, information validity, accuracy and communication are essential when planning policies and interventions. Despite the large sample size in this study, the online recruitment strategy may have limited respondent variety. Nevertheless, the participants represented all demographic groups in the country.

\section{CONCLUSIONS}

Similar engagement with COVID-19 pandemic information was observed across different demographic groups in Nigeria. Younger people preferred Twitter social media platform, considered international organizations more responsible for information accuracy, and believed individuals had a part to play. Despite low trust in the accuracy of information received, people still disseminated it widely, and a few experimented with some protection and treatment suggestions. To ensure appropriate responses to credible COVID-19 public health measures, particularly vaccination, there is a need to support social media engagement using risk awareness strategies to reduce misinformation and vaccine hesitancy.

\section{REFERENCES}

1. Dowd JB, Andriano L, Brazel DM, et al. Demographic science aids in understanding the spread and fatality rates of COVID-19. Proc Natl Acad Sci U S A. 2020;117(18):96969698. doi:10.1073/pnas.2004911117

2. Neiger BL, Thackeray R, Van Wagenen SA, et al. Use of Social Media in Health Promotion: Purposes, Key Performance Indicators, and Evaluation Metrics. Health Promot Pract. 2012;13(2):159-164. doi:10.1177/1524839911433467

3. Stecula DA, Kuru O, Jamieson KH. How Trust in Experts and Media Use Affect Acceptance of Common Anti-Vaccination Claims. Harv Kennedy Sch Misinformation Rev. 2020;1(1):111. doi:10.37016/mr-2020-007

4. U.S. Department of Health and Human Services, Centers for Disease Control and Prevention. The Health Communicator's Social Media Toolkit. U.S. Department of Health and Human Services, Centers for Disease Control and Prevention, Office of the Associate Director of Communication. July 2011. CS215469-A. Accessed November 28, 2020. https:// www.cdc.gov/healthcommunication/toolstemplates / socialmediatoolkit_bm.pdf

5. Ogoina D. Behavioural and emotional responses to the 2014 Ebola outbreak in Nigeria: a narrative review. Int Health. 2016;8(1):5-12. doi:10.1093/inthealth/ihv065

6. Metzger MJ, Flanagin AJ. Digital Media and Youth: Unparalleled Opportunity and Unprecedented Responsibility. In: Metzger MJ, Flanagin AJ, eds. Digital Media, Youth, and Credibility. The John D. and Catherine T. MacArthur Foundation Series on Digital Media and Learning. The MIT Press; 2008:5-28. doi:10.1162/dmal.9780262562324.005

7. Chukwuorji JC, Iorfa SK. Commentary on the Coronavirus 
Pandemic: Nigeria. Psychol Trauma. 2020;12(S1):S188-S190. doi: $10.1037 / \operatorname{tra} 0000786$

8. Busari S, Adebayo B. Nigeria records chloroquine poisoning after Trump endorses it for coronavirus treatment. CNN. Updated March 23, 2020. Accessed November 19, 2020. https://edition.cnn.com/2020/03/23/africa/chloroquinetrump-nigeria-intl/index.html

9. Giustini D, Ali SM, Fraser M, Kamel Boulos MN. Effective uses of social media in public health and medicine: a systematic review of systematic reviews. Online J Public Health Inform. 2018;10(2):e215. doi:10.5210/ojphi.v10i2.8270

10. Papageorge NW, Zahn MV, Belot M, et al.Socio-Demographic Factors Associated with Self-Protecting Behavior during the COVID-19 Pandemic. IZA - Institute of Labor Economics; 2020. IZA DP No. 13333. June 2020. Accessed April 17, 2021. http://ftp.iza.org/dp13333.pdf

11.Wilson SL, Wiysonge C. Social media and vaccine hesitancy. BMJ Glob Health. 2020;5(10):e004206. doi:10.1136/bmjgh-2020-004206

12. Iorfa SK, Ottu IFA, Oguntayo R, et al. COVID-19 Knowledge, Risk Perception, and Precautionary Behavior Among Nigerians: A Moderated Mediation Approach. Front Psychol. 2020;11:566773. doi:10.3389/fpsyg.2020.566773

13. Moran KR, Del Valle SY. A Meta-Analysis of the Association between Gender and Protective Behaviors in Response to Respiratory Epidemics and Pandemics. PLoS One. 2016;11(10):e0164541. doi:10.1371/journal.pone.0164541

14. Heldman AB, Schindelar J, Weaver JB. Social Media Engagement and Public Health Communication: Implications for Public Health Organizations Being Truly "Social". Public Health Rev. 2013;35(1):13. doi:10.1007/BF03391698

15. Ladan A, Haruna B, Madu AU. COVID-19 Pandemic and Social Media News in Nigeria: The Role of Libraries and Library Associations in Information Dissemination. International Journal of Innovation and Research in Educational Sciences. 2020;7(2):2349-5219. Accessed April 17, 2021. https:// www.ijires.org/administrator/components/com_jresearch/ files/publications/IJIRES_1718_FINAL.pdf

\section{ACKNOWLEDGEMENTS}

We thank the public who participated in our study. We also greatly appreciate the support of Chinedu Anarado for his contribution to the development and promotion of survey tool.

\section{CONFLICTS OF INTEREST}

The authors have completed and submitted the ICMJE Form for Disclosure of Potential Conflicts of Interest and none was reported.

\section{FUNDING}

There was no source of funding for this research.

ETHICAL APPROVAL AND INFORMED CONSENT

A cross-sectional online survey was used to collect data. Participation was voluntary. Ethical approval was obtained from the University of Hertfordshire (HUM/SF/UH/04647).

\section{DATA AVAILABILITY}

The data supporting this research is available from the authors on reasonable request.

\section{AUTHORS' CONTRIBUTIONS}

Both authors contributed to the development of the study, analysis and writing of the publication.

\section{PROVENANCE AND PEER REVIEW}

Not commissioned; externally peer reviewed. 